\title{
Tonality of Low-Frequency Synthesized Piano Tones
}

\author{
Lola L. Cuddy
}

Queen's University

Frank A. Russo

Ryerson University

\section{Alexander Galembo}

Russian Academy of Sciences

digital.library.ryerson.ca/object/153

Please Cite:

Cuddy, L. L., Russo, F. A., \& Galembo, A. (2014). Tonality of low-frequency synthesized piano tones. Archives of Acoustics, 32(3), 541-550.

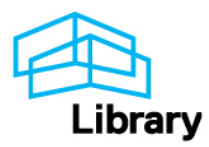




\title{
TONALITY OF LOW-FREQUENCY SYNTHESIZED PIANO TONES
}

\author{
Lola L. CUDDY ${ }^{(1)}$, Frank A. RUSSO ${ }^{(2)}$, Alexander GALEMBO ${ }^{(3)}$ \\ ${ }^{(1)}$ Queen's University \\ Department of Psychology, Kingston, Canada \\ e-mail: Lola.Cuddy@queensu.ca \\ ${ }^{(2)}$ Ryerson University \\ Department of Psychology, Toronto, Canada \\ e-mail: russo@ryerson.ca \\ ${ }^{(3)}$ Russian Academy of Sciences \\ Setchenov Institute of Evolutionary Physiology \\ and Biochemistry, St. Petersburg, Russia
}

(received May 15, 2007; accepted June 6, 2007)

\begin{abstract}
The influences of inharmonicity and bandwidth on sensitivity to tonality in the lowfrequency range ( $A_{0}$ to $\left.\mathrm{G} \#_{1}\right)$ were tested in a listening experiment. Participants were presented a key-defining context (do-mi-do-so) and were asked to rate the goodness of fit of probe tones to the context. Probe tones were the 12 tones of the chromatic scale beginning on do. The set of 12 ratings, called the probe-tone profile, was compared to an established standardized profile for the Western tonal hierarchy. Prior research employing this method with real (sampled) piano tones has suggested that sensitivity to tonality is influenced by inharmonicity, particularly in the lowest octaves of the piano where inharmonicity levels are substantially above the detection threshold. In the present experiment, sensitivity to tonality was tested using synthesized piano-like tones that were either harmonic or inharmonic. Participants were tested in either a broadband (no filtering) or low-pass (low-pass filtered at $1 \mathrm{KHz}$ ) condition. Sensitivity to tonality was highest in the broadband harmonic condition followed by the broadband inharmonic condition. No sensitivity to tonality was found for the low-pass conditions; rather, for both harmonic and inharmonic tones, participants rated probe tones as increasingly good fit as pitch distance from do decreased.
\end{abstract}

Keywords: musical pitch, tonality, probe-tone technique, inharmonicity, piano acoustics.

\section{Introduction}

This article addresses musical pitch in low-frequency, synthesized, piano-like tones. Musical pitch, in the tonal-harmonic system, refers to judgments of intervals, scale degrees, and key [1]. In particular, we are concerned with sensitivity to tonality, which 
is the perception of pitch relations according to a specified hierarchy of importance or salience of tones around a reference tone [1-4]. The diatonic system of the Western Harmonic idiom contains a four-level hierarchy of stability. The highest stability is associated with the tonic tone (do), followed by nontonic triad tones (mi, so), followed by nontriadic tones (re, fa, la, ti), and finally the nonscale tones.

Listener's sensitivity to tonality may be assessed through the probe-tone technique, a technique adopted in the present study. The technique involves presenting a musical context followed by a probe-tone, one of the 12 degrees of the chromatic scale. The listener is asked to rate the goodness-of-fit of the probe tone to the context. The set of 12 ratings is called the probe tone profile. KRUMHANSL and KESSLER produced standardized profiles for the major and minor keys by averaging probe-tone ratings across listeners and across several key-defining contexts [5]. The standardized profiles corresponded to the hierarchical description of tonality above and may be used as a referent against which to compare profiles obtained under other varying experimental conditions. The correlation between an obtained profile and a standardized profile yields a recovery score. A high score is considered evidence of strong recovery of the tonal hierarchy.

Listeners experienced with the music of Western culture produce strong recovery of the tonal hierarchy with different stimulus contexts and various instrumental and synthetic timbres. However, various observations support the notion that sensitivity to tonality may be compromised in the low-frequency range of musical instruments. Loss of sensitivity may be attributable to psychoacoustic factors affecting tone differentiation in the low-frequency range and may have implications for instrument design, especially for the possibilities attached to instrumental tone synthesis.

Direct evidence for reduced sensitivity is scarce but critical. First, RAKOWSKI and MISKIEWICZ have argued that pitch discrimination for low musical tones is best modeled by the rising difference limens found for pure-tones below $200 \mathrm{~Hz}$ [6]. They report three studies (one their own) showing that pure-tone discrimination limens in the lowfrequency range reach nearly a semitone at the nominal frequency of the lowest piano tone $\mathrm{A}_{0}, f_{0}=27.5 \mathrm{~Hz}$. Rakowski and Miskiewicz argue that the pitch of a low musical tone is that of the residue evoked by a periodic sound $[7,8]$ and that the neural discharge evoked is identical to that evoked by a pure tone. Given Rakowski's argument, it follows that such poor discrimination would have consequences for musical tonality.

Second, Patterson and colleagues have reported that the lower limit of melodic pitch for harmonic complex tones is a value close to the frequency at which the difference limen for pure tones rises to a semitone $[9,10]$. Their task involved the comparison of two four-tone melodies the second of which might or might not include a change of one semitone in one of the tones. The authors note, however, that the task does not require the judgment of musical pitch relations and thus the relevance to musical pitch processing is debatable. Nevertheless, the findings suggest that sensitivity to tonality may be compromised in the low-frequency range as the lower limit of melodic pitch is approached. 
Third, the inharmonicity of many musical instruments (i.e., progressive stretching of higher partials resulting in aperiodicity) may have adverse consequences on sensitivity to tonality. Instruments involving struck or plucked strings - including the piano possess inharmonicity resulting from deviations from ideal string elasticity [11]. For the piano, inharmonicity is above threshold in the lower range of the tessitura [12]. RUSSO, CUdDy, GALEMBO and THOMPSON found that sensitivity to tonality for real (sampled) piano tones was impoverished in the lowest octaves compared to sensitivity in the midrange of the piano [13]. A regression model showed that recovery scores across the tessitura were successfully predicted by two quantified measures - suprathreshold inharmonicity and pitch salience.

Suprathreshold inharmonicity was quantified as the difference between threshold inharmonicity [12] and the measured inharmonicity of the piano tones. Pitch salience values were calculated according to TERHARDT, STOLL and SEEWAN's model [14]. According to this model, pitch salience depends on spectral dominance, masking, and subharmonic co-incidence. It does not take into account the threshold of inharmonicity. Despite the success of the model in fitting the observed data, it may be noted that factors co-varying with pitch salience - such as the frequency of occurrence of tones in keyboard music - could also account for the data.

In the present experiment, we follow the study by RUSSO et al. Their main experiment tested recovery of the tonal hierarchy for differing levels of inharmonicity but did not compare performance directly for inharmonic and harmonic tones. Here we report a direct comparison of sensitivity to tonality for synthesized low-frequency piano-like tones that either possessed or did not possess inharmonicity. Tones were broadband with 73 components and had the spectral and temporal envelope of a real piano tone. We expected that eliminating inharmonicity would yield an improvement in sensitivity to tonality, an expectation with which differing models of pitch perception would agree. Temporal models suggest that pitch perception would benefit from the unresolved higher-order harmonic partials that determine the residue pitch $[15,16]$. Spectral models that invoke pattern matching predict more reliable pitch perception when harmonic as opposed to inharmonic partials are available [17, 18].

The outcome is not obvious, however. Eliminating inharmonicity alters the pianolike timbre associated with inharmonicity to a less familiar timbre. Pitch salience will still be low. If familiarity and pitch salience play a greater role than inharmonicity, then the possible advantage of eliminating inharmonicity may be slight (see also [19]).

A second manipulation involved the bandwidth of the synthesized tones. The broadband harmonic and inharmonic tones were low-pass filtered at $1 \mathrm{KHz}$. and we compared recovery of the tonal hierarchy for filtered as opposed to broadband tones. The application of the filter allowed us to assess the role of upper unresolved partials in the recovery of the tonal hierarchy for low-frequency tones, a role that was not implemented in the derivation of the pitch salience predictor. Several outcomes of applying the filter are possible. 
First, the upper unresolved partials may contribute to the negative effects of inharmonicity on sensitivity to tonality, as the stretching of the partials of the inharmonic piano tone increases progressively with increasing partial number $[19,20]$. Thus, removing these partials may decrease suprathreshold inharmonicity and yield stronger recovery of the tonal hierarchy. Second, for harmonic tones at least, if the unresolved partials reinforce the periodicity of the fundamental [6], their removal may weaken the recovery of the tonal hierarchy. Third, should the primary support for recovery of the tonal hierarchy be the partials in the dominant region for the residue $[14,17]$, the filter will have little or no effect on the recovery of the tonal hierarchy.

The experimental design comprised four experimental conditions created by crossing the factors of inharmonicity (harmonic vs inharmonic) and bandwidth (broadband or low-pass). Inharmonicity was assessed within-subjects; bandwidth was assessed betweensubjects. The probe-tone technique was applied in all conditions.

\section{Method}

\subsection{Participants}

Twenty-four musically trained participants ( 20 females and 4 males, mean age 20.2 years, range 18-24 years) were recruited from the Queen's University community. Mean years of music instruction was 10.5 , range $8-15$ years. Participants typically had experience with 2 instruments and continued activity in music beyond casual listening. No participant reported having absolute pitch or abnormal hearing. All participants were given course credit for their participation.

\subsection{Pretest materials and procedure}

Participants were pre-tested to provide assurance that they understood the task and could recover the standardized tonal hierarchy under optimal test conditions. Tones for the pretest were 12 real (sampled) piano tones produced by a Roland Sound Canvas MIDI module under the control of a Macintosh Power PC. Tones spanned the range $\mathrm{C}_{4}$ $\left(f_{0}=261.6 \mathrm{~Hz}\right)$ to $\mathrm{B}_{4}\left(f_{0}=493.9 \mathrm{~Hz}\right)$. Tones were presented in a sound-attenuated chamber and were delivered over Sennheiser HD280 headphones.

Each participant received a block of 12 trials. For each trial the stimulus context was a melodic major triad - do, mi, do, so. Tones of the context were presented in contiguous sequence with duration of each tone $.33 \mathrm{~s}$. The context was followed by a 1-s silent gap and then followed by a .33-s probe tone. The probe tone was randomly selected without replacement from the 12 notes of the chromatic scale beginning on do. The order was independently randomized for each participant. The task was to rate the degree to which the probe tone fit the context on a 7-point scale that ranged from 
"Fits very poorly" to "Fits very well". Participants were encouraged to assess the probe tone with respect to the entire 4-tone context rather than on how well the probe tone continued the melody.

\subsection{Main experiment materials and procedure}

Twelve tones were computer-synthesized in the Soundswell signal workstation running an additive synthesis program [21]. Tones were generated with a $44.1 \mathrm{KHz}$ sampling rate and spanned the range $\mathrm{A}_{0}\left(f_{0}=27.5 \mathrm{~Hz}\right)$ to $\mathrm{G}_{1}^{\#}\left(f_{0}=51.9 \mathrm{~Hz}\right)$. All tones were tuned to equal temperament based on the $\mathrm{A}_{4}(440 \mathrm{~Hz})$ standard.

For harmonic tones, partial frequencies were integer multiples of the fundamental frequency. For inharmonic tones, partial frequencies adhered to the classic formula describing inharmonicity in a stringed instrument

$$
f_{n}=n f_{0} \sqrt{1+B n^{2}}
$$

where $f_{n}$ is the frequency of the $n$-th partial, $n$ is the partial number, $f_{0}$ is the fundamental frequency for an ideally flexible string, and $B$ is the inharmonicity coefficient, determined by the string dimensions, material, and design. The inharmonicity coeefficients followed the power function $\left(B=0.013 f_{0}^{-1}\right)$ [22] with values ranging from 0.00048 for $A_{0}$ to 0.00026 for $G_{1}^{\#}$. This range is above the threshold values for inharmoncity [12] and within the range of observed values for acoustic pianos [11, 23, 24]. In the broadband condition, each tone had 73 components, and a piano-like spectral and temporal envelope. In the low-pass condition, the broadband tones were filtered by a low-pass $1 \mathrm{KHz}$ filter in the Soundswell signal workstation. Slope of the cutoff was $-6 \mathrm{~dB}$ per octave. The number of partials retained after filtering decreased with increasing fundamental frequency. For the lowest tone $\left(\mathrm{A}_{0}\right)$, partials 1 through 36 were retained. For the highest tone $\left(\mathrm{G}_{1}^{\#}\right)$, partials 1 through 19 were retained. Filtering resulted in an audible change of timbre.

Loudness of all tones was equalized based on average loudness matching by three expert listeners. Intensity of the standard tone $\left(\mathrm{A}_{0}\right)$ for loudness matching was $70 \mathrm{~dB}$ SPL for both broadband and low-pass conditions.

Participants were randomly assigned to either the broadband or low-pass condition. All participants in both conditions were tested under both the harmonic and inharmonic presentation conditions. The design of the test trials was identical to the pretest. Each participant received 4 blocks of 12 trials, i.e., 48 trials. Two blocks of trials contained harmonic tones and two blocks contained inharmonic tones. Harmonic $(\mathrm{H})$ and inharmonic blocks (I) were presented in alternating sequence with the order of blocks counterbalanced across participants (i.e., HIHI or IHIH). 


\section{Results}

\subsection{Pretest results}

All participants successfully recovered the tonal hierarchy in the pretest - that is, the probe tone ratings for each participant correlated significantly with the values of the standardized tonal hierarchy [5]. The mean pretest recovery score for the participants in the broadband condition was $.83(\mathrm{SE}=.02)$ and for participants in the low-pass condition was $.82(\mathrm{SE}=.03)$.

\subsection{Main experiment results}

Figures $1 \mathrm{a}$ and $1 \mathrm{~b}$, representing the broadband and low-pass groups respectively, show mean probe tone profiles obtained in both the harmonic and inharmonic conditions along with the standardized major-key profile for comparison. Probe tones from do $\left(\mathrm{A}_{0}\right)$ to ti $\left(\mathrm{GH}_{1}\right)$ are represented on the $x$-axis; mean rating across participants is represented on the $y$-axis.

For the broadband condition (Fig. 1a), close correspondence of the harmonic-condition profile with the standardized profile is readily apparent, $r(10)=.94, p<.001$. The inharmonic- condition profile also yielded correspondence with the standardized profile, $r(10)=.67, p<.05$, but the correlation was significantly lower than the harmonic condition, $z=1.97, p<.05$.

For the low-pass condition, neither the harmonic-condition nor the inharmonic- condition profile was significantly correlated with the standardized profile, $r(10)=.29$ and .55 , both $p>.05$, for the harmonic and inharmonic conditions, respectively. The correlations were not significantly different, $z=-.68, p=.50$.

Recovery scores for each participant were subjected to analysis of variance with bandwidth as the between-subject factor and harmonicity condition and block as the within-subject factors. As expected from the correlational analysis above, there was a significant interaction between bandwidth and harmonicity, $F(1,22)=23.70, p<$ .0001 . Orthogonal contrasts revealed that within the broadband condition, recovery scores were significantly higher in the harmonic condition $(\mathrm{M}=.61, \mathrm{SE}=.06)$ than in the inharmonic condition $(\mathrm{M}=.17, \mathrm{SE}=.11), F(1,22)=25.27, p<.0001$. Within the low-pass condition, recovery scores were not significantly different between the harmonic condition $(\mathrm{M}=.07, \mathrm{SE}=.08)$ and the inharmonic condition $(\mathrm{M}=.23$, $\mathrm{SE}=.07), F(1,22)=3.45$. The main effect of block and interactions with block were not significant.

Despite the high recovery for the broadband harmonic condition, it may be noted for the 12 participants in that condition, that the mean recovery score was nevertheless significantly lower than the mean recovery in the pretest, $t(11)=3.61, p<.01$.

Further examination of Fig. 1b, low-pass conditions, reveals a linear trend with probe tone ratings decreasing as the nominal frequency of the probe tone increases. 
a)

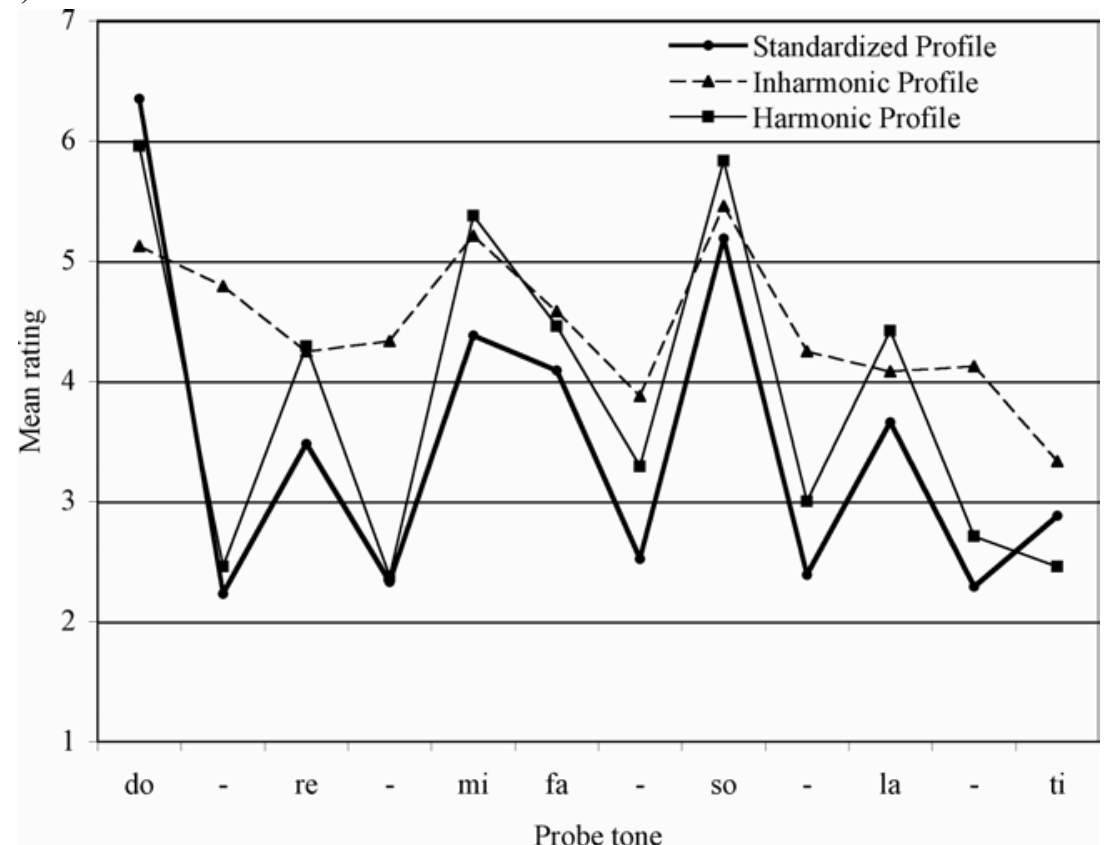

b)

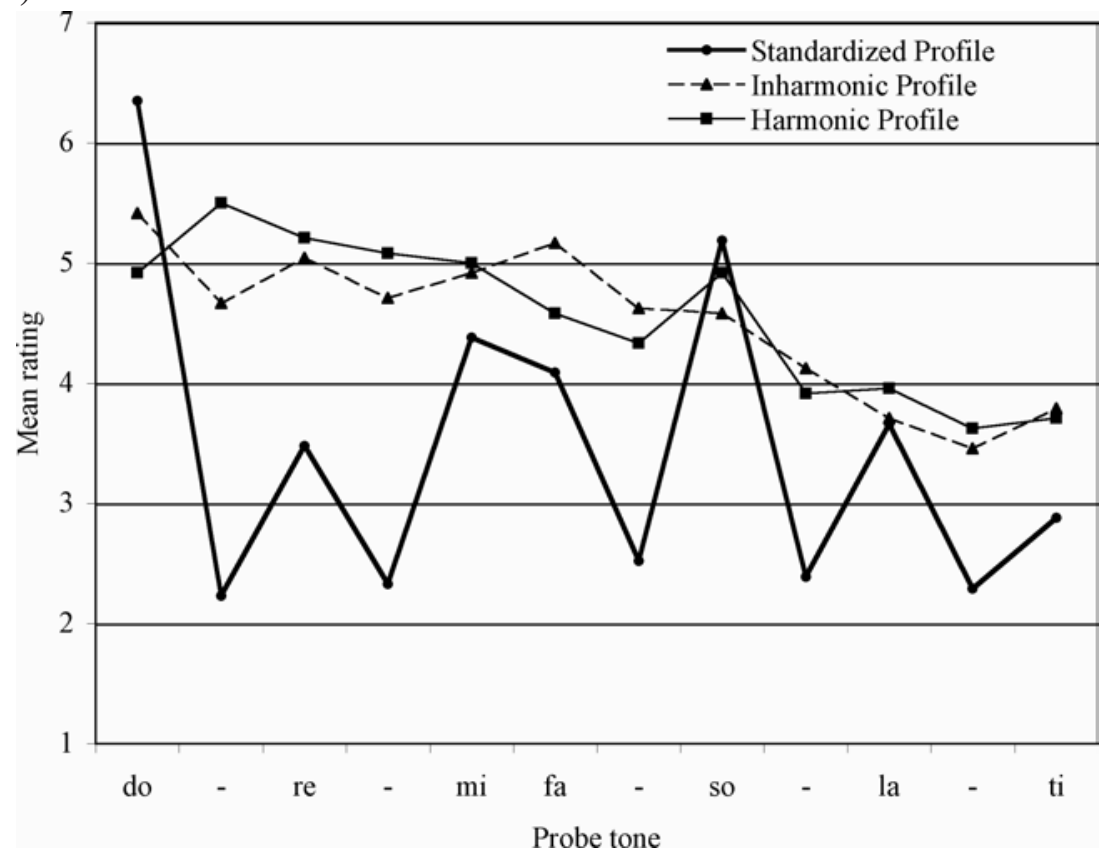

Fig. 1. Mean probe-tone profiles for the harmonic and inharmonic conditions are shown in each panel. Figure 1a represents the broadband condition and Figure $1 \mathrm{~b}$ represents the low-pass condition. The standardized major-key profile (KRUMHANSL and KESSLER [5]) is included in each panel for comparison. 
The correlation of mean ratings with linearity was $r(10)=.90, p<.001$, for the harmonic condition and $r(10)=.87, p<.001$, for the inharmonic condition. Analysis of variance of participants' ratings in the low-pass condition, averaged across harmonicity, also yielded a significant linear trend, $F(1,11)=10.07, p<.01$. No significant linear trend was found for broadband conditions.

\section{Discussion}

For the four conditions tested, the tonal hierarchy was reliably recovered in the harmonic broadband condition, less reliably recovered in the inharmonic broadband condition, and not recovered in the harmonic and inharmonic low-pass conditions. In other words, sensitivity to tonality was most evident for a broad harmonic spectrum and was adversely affected by the low-pass filtering of that spectrum at $1 \mathrm{kHz}$. As we found previously [13], suprathreshold inharmonicity had adverse effects on recovery of the tonal hierarchy. However, our expectation that removing the upper partials of an inharmonic spectrum might reduce the adverse effect of inharmonicity was not borne out. In fact, the effect of removing the upper partials was to remove evidence of recovery of the tonal hierarchy.

The findings indicate that higher-order unresolved partials beyond the dominant region may be crucial to the formation of musical pitch relations. Such a finding is consistent with temporal models of pitch perception that posit the importance of unresolved partials in the formation of residue pitch. We leave to future research the modification of our earlier regression model [13] to incorporate the contribution of unresolved partials throughout the tessitura.

Although the tonal hierarchy was not recovered in the low-pass conditions, there was significant differentiation among the probe tones. Differentiation took the form of a linear function with probe tone ratings negatively related to pitch height, or equivalently, the pitch distance from the do of the stimulus context triad. Probe tones were rated decreasingly lower (poorer "fit" to the context) as the distance from do increased. In the terms of the original helical model of musical pitch [25], pitch-height (low-tohigh pitch) differentiation was preserved in the filtered conditions. Pitch chroma, which represents the musical location of tones within the octave, and which in later models gives rise to the tonal hierarchy, was lost.

Our results may have implications for the emerging area of sound synthesis by physical modeling. This type of sound synthesis has the potential to provide unprecedented realism compared to conventional sampling or synthesis methods. However, the approach is limited in real time applications because it is computationally intensive. In the case of inharmonicity, several researchers have noted that important computational savings may be obtained by omitting the inharmonicity layer of sound synthesis in high frequency tones where it should have minimal consequence for tone quality [12, 26]. Our results suggest that reducing inharmonicity may also be beneficial for low frequency tones, especially in applications where tonal sensitivity is deemed important. 
A natural concern is whether the timbral change resulting from the omission of inharmonicity in low-frequency piano tones would affect judgments of piano-tone quality [24]. The concern is probably unfounded. Inharmonicity is less important in defining the piano timbre than is the co-varying factor of spectral bandwidth [19]. A broad spectral bandwidth along with harmonicity should not only enhance sensitivity to tonality in the low-frequency range but also produce an agreeable piano sound.

\section{Acknowledgments}

Research was funded by a Discovery Grant to L. L. Cuddy from the Natural Sciences and Engineering Council of Canada and a NATO Postdoctoral Science Fellowship to A. Galembo.

\section{References}

[1] Krumhansl C. L., Cognitive foundations of musical pitch, Oxford University Press, New York 1990.

[2] LeRdahl F., Tonal pitch space, Oxford University Press, New York 2001.

[3] PIston W., Harmony, 5th ed., W.W. Norton \& Company, Inc, New York 1987.

[4] Krumhansl C. L., Rhythm and pitch in music cognition, Psychological Bulletin, 126, 1, 159-179 (2000).

[5] Krumhansl C. L., Kessler E. J., Tracing the dynamic changes in perceived tonal organization in a spatial representation of musical keys, Psychological Review, 89, 4, 334-368 (1982).

[6] Rakowski A., Miskiewicz A., Pitch discrimination of low-frequency tones, Proceedings of the 7-th International Conference on Music Perception and Cognition, Sydney, 538-540, 2002.

[7] Ritsma R. J., Existence region of the tonal residue: Part I, Journal of the Acoustical Society of America, 34, 9A, 1224-1229 (1962).

[8] Ritsma R. J., Existence region of the tonal residue: Part II, Journal of the Acoustical Society of America, 35, 8, 1241-1245 (1963).

[9] Krumbholtz K., Patterson R. D., Pressnitzer D., The lower limit of pitch as determined by rate discrimination, Journal of the Acoustical Society of America, 108, 3, 1170-1180 (2000).

[10] Pressnitzer D., Patterson R. D., Krumbholz K., The lower limit of melodic pitch, Journal of the Acoustical Society of America, 109, 5, 2074-2084 (2001).

[11] FlETCHER H., Normal vibration frequencies of a stiff piano string, Journal of the Acoustical Society of America, 36, 1, 203-209 (1964).

[12] JärVeläinen H., Valimaki V., Karjalainen M., Audibility of the timbral effects of inharmonicity in stringed instrument tones, Acoustics Research Letters Online, 2, 3, 79-84 (2001).

[13] Russo F. A., Cuddy L. L., Galembo A., Thompson W. F., Sensitivity to tonality across the pitch range, Perception, 36, in press. 
[14] Terhardt E., Stoll G., Seewann M., Pitch of complex signals according to virtual-pitch theory: Test, examples, and predictions, Journal of the Acoustical Society of America, 71, 3, 671-678 (1982).

[15] PATterson R. D., The effects of relative phase and the number of components on residue pitch, Journal of the Acoustical Society of America, 53, 6, 1565-1572 (1973).

[16] Schouten J. F., Ritsma R. J., Cardozo B. L., Pitch of the residue, Journal of the Acoustical Society of America, 34, 9B, 1418-1424 (1962).

[17] Terhardt E., Pitch, consonance and harmony, Journal of the Acoustical Society of America, 55, 5, 1061-1069 (1974).

[18] Wightman F. L., The pattern-transformation model of pitch, Journal of the Acoustical Society of America, 54, 2, 407-416 (1973).

[19] Galembo A., Askenfelt A., Cuddy L. L., Russo F. A., Perceptual significance of inharmonicity and spectral envelope in the piano bass range, Acta Acustica, 90, 3, 528-536 (2004).

[20] Schuck O. H., Young R. W., Observations on the vibrations of piano strings, Journal of the Acoustical Society of America, 15, 1, 1-11 (1943).

[21] NyVAlla, Soundswell signal workstation, Stockholm, Sweden, 1997.

[22] Rasch A., Heetvelt V., String inharmonicity and piano tuning, Music Perception, 3, 2, 171-190 (1985).

[23] Conklin H. A., Generation of partials due to nonlinear mixing in stringed instruments, Journal of the Acoustical Society of America, 105, 1, 536-545 (1999).

[24] Fletcher H., Blackham E. D., Stratton R., Quality of piano tones, Journal of the Acoustical Society of America, 34, 6, 749-761 (1962).

[25] SHEPARD R. N., Geometrical approximations to the structure of musical pitch, Psychological Review, 89, 4, 305-33 (1982).

[26] Rocchesso D., SCAlCon F., Bandwidth of perceived inharmonicity for physical modeling of dispersive strings, IEEE Transactions in Speech and Audio Processing, 7, 5, 597-601 (1999). 